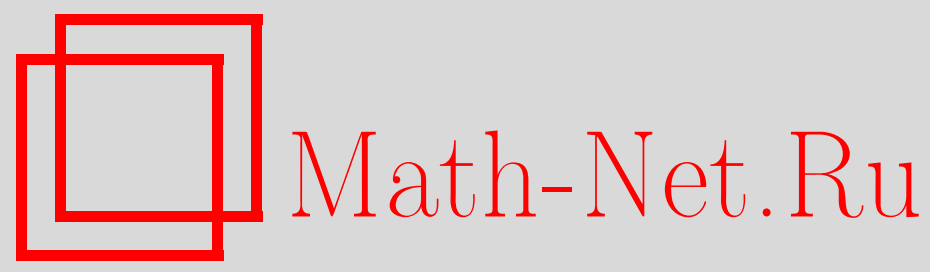

М. А. Ольшанский, Е. В. Чижонков, О наилучшей константе в inf-sup-условии для вытянутых прямоугольных областей, Матем. заметки, 2000, том 67, выпуск 3, 387-396

DOI: https://doi.org/10.4213/mzm852

Использование Общероссийского математического портала Math-Net.Ru подразумевает, что вы прочитали и согласны с пользовательским соглашением http://www.mathnet.ru/rus/agreement

Параметры загрузки:

IP: 54.157 .27 .8

26 апреля 2023 г., $14: 38: 32$

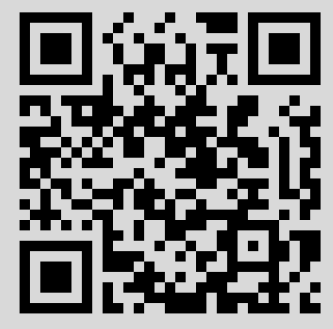


УДК 517.984 .5

\title{
О НАИЛУЧШЕЙ КОНСТАНТЕ В inf-sup-УСЛОВИИ ДЛЯ ВЫТЯНУТЫХ ПРЯМОУГОЛЬНЫХ ОБЛАСТЕЙ
}

\author{
М.А. Ольшанский, Е. В. Чижонков
}

При анализе сходимости приближенных решений уравнений динамики жидкости к точньм часто используется неравенство Ладыженской-Бабушки-Брецци (inf-supусловие). Входящая в него константа зависит от формы области и определяет эффективность различных алгоритмов. В работе получена ее асимптотика и двусторонние оценки в прямоугольных областях. Для этого используется новый способ получения оценок собственных значений некоторой спектральой задачи с седловым оператором Стокса.

Библиографоия: 13 названий.

1. Введение. Рассмотрим первую краевую задачу для уравнений Стокса в переменных скорость-давление

$$
\begin{aligned}
-\Delta \boldsymbol{u}+\operatorname{grad} p=f & \text { в } \Omega, \\
\operatorname{div} \boldsymbol{u}=0 & \text { в } \Omega, \\
\boldsymbol{u}=0 & \text { на } \partial \Omega .
\end{aligned}
$$

Уравнения (1) описьвают движение вязкой несжимаемой однородной и изотропной жидкости при малых скоростях под воздействием внешних сил. Неизвестньпи здесь являются вектор-функция $\boldsymbol{u}=\left(u_{1}(x), \ldots, u_{s}(x)\right)$ (скорость жидкости) и скалярная функция $p=p(x)$ (давление). Нулевые граничные условия означают, как правило, что движение происходит в некотором неподвижном объеме с твердыми стенками, полностью заполненном жидкостью. Описанная модельная задача имеет самое разнообразное применение в вычислительной гидродинамике: начиная от построения дискретных аналогов и заканчивая сравнением эффективности численных алгоритмов.

При обосновании корректности постановки и приближенном решении (1) важную роль играет величина $\gamma=\gamma(\Omega)$, зависящая только от области $\Omega \subset \mathbb{R}^{s}(s=2,3)$ и определяемая равенством

$$
\inf _{p \in P} \sup _{\boldsymbol{u} \in \boldsymbol{U}} \frac{|(p, \operatorname{div} \boldsymbol{u})|}{\|\boldsymbol{u}\|_{\boldsymbol{U}}\|p\|_{P}}=\gamma .
$$

Здесь и далее

$$
\begin{gathered}
P=\left\{q \mid q \in L^{2}(\Omega), \int_{\Omega} q d \Omega=0\right\}, \quad \boldsymbol{U}=\left(\stackrel{\circ}{W}_{2}^{1}(\Omega)\right)^{s} \\
\|\boldsymbol{u}\|_{\boldsymbol{U}}=(\nabla \boldsymbol{u}, \nabla \boldsymbol{u})^{1 / 2}, \quad\|p\|_{P}=(p, p)^{1 / 2}, \quad(\phi, \psi)=\int_{\Omega} \phi \psi d \Omega .
\end{gathered}
$$

Работа выполнена при частичной финансовой поддержке Российского фонда фундаменталных исследований, гранты № 96-01-01254 и № 96-01-01394. 
Действительно, для ограниченных связных областей с кусочно-липшицевой границей, известно (см., например, [1], [2]), что $\gamma>0$, и для обобщенного решения задачи (1) $(\boldsymbol{u}, p) \in \boldsymbol{U} \times P$ справедливы следующие априорные оценки:

$$
\|p\|_{P} \leqslant 2 \gamma^{-1}\|\boldsymbol{f}\|_{\boldsymbol{U}^{-1}}, \quad\|\boldsymbol{u}\|_{\boldsymbol{U}} \leqslant\|\boldsymbol{f}\|_{\boldsymbol{U}^{-1}}, \quad \text { где }\|\boldsymbol{f}\|_{\boldsymbol{U}^{-1}}=\sup _{\boldsymbol{u} \in \boldsymbol{U}} \frac{|(\boldsymbol{f}, \boldsymbol{u})|}{\|\boldsymbol{u}\|_{\boldsymbol{U}}} .
$$

Кроме того, считающийся в настоящее время одним из наиболее эффективных для численного решения задачи Стокса метод Удзавы - сопряженных градиентов имеет асимптотический показатель скорости сходимости [3]

$$
\kappa(\gamma)=\frac{1-\gamma}{1+\gamma}
$$

Зависимость подобного типа от $\gamma$ установлена для скорости сходимости и других методов для (1), в которьх основным элементом является многократное решение уравнения Пуассона (см., например, [4], [5]).

Отметим также, что константа $\gamma$ тесно связана со своим дискретньг аналогом $\gamma_{h}$ (см., например, [2]). Последняя широко известна как константа из LBB или inf-sup-ycловия и имеет принципиальное значение при анализе схем метода конечных элементов для (1).

В связи с разнообразием задач вычислительной гидродинамики и широким распространением подходов к решению, основанных на разбиении исходной области на ряд более "простых" подобластей, представляется интересным анализ зависимости значений $\gamma$ в (2) от геометрических характеристик области $\Omega$.

Ранее предпринимались попытки определить величину $\gamma$ (или тесно связанную с ней нижнюю границу конечнократного спектра Коссера [6]), но это всегда было связано с формой области $\Omega$, допускающей применение метода разделения переменных (например, шаровой слой [7], кольцо [8]). В этом случае, решая аналитически некоторую вспомогательную спектральную задачу для системы обыкновенных дифференциальных уравнений, можно определить нижнюю границу собственных значений, откуда получается искомое значение $\gamma$.

В настояшей работе устанавливается справедливость следующего утверждения: пусть область $\Omega$ имеет прямоугольную форму

$$
\Omega=\left\{\left(x_{1}, x_{2}\right) \mid 0<x_{i}<L_{i}, i=1,2\right\}
$$

и $\ell=\max \left(L_{1} / L_{2}, L_{2} / L_{1}\right)$; тогда при $\ell \gg 1$ имеет место асимптотическое представление $\gamma=O\left(\ell^{-1}\right)$. Следует отметить, что в данном случае описанный вьше подход неприменим, и точное значение $\gamma$ при фиксированном $\ell$ определить не удается, хотя содержательные оценки сверху и снизу также получены.

2. Дополнения по Шуру. Перепишем задачу (1) в следующем равносильном виде:

$$
\begin{gathered}
\boldsymbol{u}=(\Delta)_{0}^{-1}(\operatorname{grad} p-\boldsymbol{f}) \\
A_{0} p=\operatorname{div}(\Delta)_{0}^{-1} \operatorname{grad} p=\operatorname{div}(\Delta)_{0}^{-1} \boldsymbol{f}
\end{gathered}
$$

где выражение $(\Delta)_{0}^{-1} \boldsymbol{g}$ для $\boldsymbol{g} \in \boldsymbol{U}^{-1}$ обозначает вектор-функцию $\boldsymbol{v} \in \boldsymbol{U}$ такую, что $\Delta \boldsymbol{v}=\boldsymbol{g}$. Оператор $A_{0}: P \rightarrow P$, как правило, называют дополнением по Шуру для 
оператора задачи (1). Его замечательные свойства [6], [9], [10]: самосопряженность, положительная определенность, дискретность спектра, наличие полной ортонормированной в $P$ счетной системы собственных функций - часто используются для анализа и обоснования методов решения задачи Стокса. Существует точка зрения [1], что методы, основанные на решении системы $A_{0} p=\varphi$, являются в определенном смысле наилучшими. В частности, к таким методам относятся различные модификации алгоритма Удзавы (или метода окаймления) [3].

Для наших целей важной является связь минимального собственного значения оператора $A_{0}$ с постоянной $\gamma$ из (2). Расмотрим следующую цепочку равенств, справедливых для произвольной функции $q \in W_{2}^{1}(\Omega) \cap P$ :

$$
\begin{aligned}
\left(A_{0} q, q\right) & =\left(\operatorname{div}(\Delta)_{0}^{-1} \operatorname{grad} q, q\right)=-\left((\Delta)_{0}^{-1} \operatorname{grad} q, \operatorname{grad} q\right) \\
& =-\left((\Delta)_{0}^{-1} \operatorname{grad} q, \Delta(\Delta)_{0}^{-1} \operatorname{grad} q\right)=\left.(-\Delta \boldsymbol{v}, \boldsymbol{v})\right|_{\boldsymbol{v}}=(\Delta)_{0}^{-1} \operatorname{grad} q \\
& =\|\boldsymbol{v}\|_{\boldsymbol{U}}^{2}=\sup _{\boldsymbol{u} \in \boldsymbol{U}} \frac{(-\Delta \boldsymbol{v}, \boldsymbol{u})^{2}}{\|\boldsymbol{u}\|_{\boldsymbol{U}}^{2}}=\sup _{\boldsymbol{u} \in \boldsymbol{U}} \frac{(\operatorname{grad} q, \boldsymbol{u})^{2}}{\|\boldsymbol{u}\|_{\boldsymbol{U}}^{2}}=\sup _{\boldsymbol{u} \in \boldsymbol{U}} \frac{(q, \operatorname{div} \boldsymbol{u})^{2}}{\|\boldsymbol{u}\|_{\boldsymbol{U}}^{2}} .
\end{aligned}
$$

Множество функций из $W_{2}^{1}(\Omega)$ всюду плотно [11] в $P$, откуда следует корректность замыкания в равенствах

$$
\left(A_{0} q, q\right)=\|\boldsymbol{v}\|_{\boldsymbol{U}}^{2}=\sup _{\boldsymbol{u} \in \boldsymbol{U}} \frac{(q, \operatorname{div} \boldsymbol{u})^{2}}{\|\boldsymbol{u}\|_{\boldsymbol{U}}^{2}} .
$$

Таким образом, равенства (3) справедливы для произвольной $q \in P$ и $\boldsymbol{v}=\Delta_{0}^{-1} \operatorname{grad} q \in \boldsymbol{U}$. В частности, из (2) и (3) следует, что

$$
\lambda_{\min }\left(A_{0}\right)=\inf _{p \in P} \frac{\left(A_{0} p, p\right)}{\|p\|_{P}^{2}}=\inf _{p \in P} \sup _{\boldsymbol{u} \in \boldsymbol{U}} \frac{(p, \operatorname{div} \boldsymbol{u})^{2}}{\|\boldsymbol{u}\|_{\boldsymbol{U}}^{2}\|p\|_{P}^{2}}=\gamma^{2},
$$

где $\lambda_{\min }$ - минимальное собственное значение оператора $A_{0}$.

Далее нам потребуются операторы $A_{p}$ и $A_{m}$, родственные $A_{0}$. Здесь имеется в виду, что они являются дополнениями по Шуру для операторов задач типа (1), но с другими краевьми условиями. Начиная с этого момента будем считать, что область $\Omega$ - прямоугольник:

$$
\Omega=\left\{\left(x_{1}, x_{2}\right) \mid 0<x_{i}<L_{i}, i=1,2\right\} .
$$

Обозначим через $A_{p}$ оператор $A_{p}: P \rightarrow P$ вида

$$
A_{p}=\operatorname{div}(\Delta)_{p}^{-1} \operatorname{grad},
$$

где выражение $(\Delta)_{p}^{-1} \boldsymbol{g}$ для $\boldsymbol{g} \in \boldsymbol{U}_{p}^{-1}$ обозначает вектор-функцию $\boldsymbol{v} \in \boldsymbol{U}_{p}$ такую, что

$$
-(\nabla \boldsymbol{v}, \nabla \boldsymbol{u})=(\boldsymbol{g}, \boldsymbol{u}) \quad \forall \boldsymbol{u} \in \boldsymbol{U}_{p},
$$

где $\boldsymbol{U}_{p}=\left\{\boldsymbol{u} \mid \boldsymbol{u} \in\left(W_{2}^{1}(\Omega)\right)^{2}, \boldsymbol{u} \cdot n=0\right.$ на $\left.\partial \Omega\right\}$. Равенство (5) является определением обобщенного решения для векторного уравнения Пуассона с краевыми условиями следующего вида:

$$
\boldsymbol{u} \cdot n=0, \quad \frac{\partial(\boldsymbol{u} \cdot \tau)}{\partial n}=0 \text { на } \partial \Omega .
$$


Здесь $n$ и $\tau$ - нормальный и касательньй векторы к $\partial \Omega$. Аналогичньм образом можно сформулировать и задачу для уравнений Стокса с такими краевыми условиями. Отметим, что, с одной стороны, $A_{p} \equiv E[12]$ ( $E$ - тождественньй оператор), а с другой - (как и в (3)) для произвольной $q \in P$ и $v=\Delta_{p}^{-1} \operatorname{grad} q$ имеем

$$
\left(A_{p} q, q\right)=\sup _{\boldsymbol{u} \in \boldsymbol{U}_{p}} \frac{(q, \operatorname{div} \boldsymbol{u})^{2}}{\|\boldsymbol{u}\|_{\boldsymbol{U}}^{2}}=\|\boldsymbol{v}\|_{\boldsymbol{U}}^{2}
$$

Из равенств (6) следует, что верхняя грань в выражении

$$
\sup _{\boldsymbol{u} \in \boldsymbol{U}_{p}} \frac{(q, \operatorname{div} \boldsymbol{u})^{2}}{\|\boldsymbol{u}\|_{\boldsymbol{U}}^{2}}
$$

достигается на функции $v=\Delta_{p}^{-1} \operatorname{grad} q$. Этот факт будет использоваться нами в дальнейшем.

Придерживаясь той же схемы, обозначим через $A_{m}$ оператор $A_{m}: P \rightarrow P$ вида

$$
A_{m}=\operatorname{div}(\Delta)_{m}^{-1} \operatorname{grad}
$$

где выражение $(\Delta)_{m}^{-1} \boldsymbol{g}$ для $\boldsymbol{g} \in \boldsymbol{U}_{m}^{-1}$ обозначает вектор-функцию $\boldsymbol{v} \in \boldsymbol{U}_{m}$ такую, что

$$
-(\nabla \boldsymbol{v}, \nabla \boldsymbol{u})=(\boldsymbol{g}, \boldsymbol{u}) \quad \forall \boldsymbol{u} \in \boldsymbol{U}_{m}
$$

где $\boldsymbol{U}_{m}=\left\{\boldsymbol{u}=\left(u_{1}, u_{2}\right) \mid \boldsymbol{u} \in\left(W_{2}^{1}(\Omega)\right)^{2}, u_{1}=0,\left(0, u_{2}\right) \cdot n=0\right.$ на $\left.\partial \Omega\right\}$. Равенство $(7)$ соответствует определению обобщенного решения для векторного уравнения Пуассона с краевыми условиями смешанного типа

$$
\begin{aligned}
\boldsymbol{u} & =0 \quad \text { при } x_{2}=0, L_{2}, \\
\boldsymbol{u} \cdot n & =0, \quad \frac{\partial(\boldsymbol{u} \cdot \tau)}{\partial n}=0 \quad \text { при } x_{1}=0, L_{1} .
\end{aligned}
$$

В данном случае также справедливо равенство для произвольной $q \in P$

$$
\left(A_{m} q, q\right)=\sup _{\boldsymbol{u} \in \boldsymbol{U}_{m}} \frac{(q, \operatorname{div} \boldsymbol{u})^{2}}{\|\boldsymbol{u}\|_{\boldsymbol{U}}^{2}} .
$$

В заключение раздела отметим, что основной результат об асимптотике $\left(\gamma \approx \ell^{-1}\right)$ следует из некоторого утверждения для минимальных собственных значений операторов $A_{i}(i=0, p, m)$. Приведем его, положив для определенности $\ell=L_{1} / L_{2}$.

УТВЕРЖДЕНИЕ. Существуют постоянные $\theta_{i}>0(i=1,2)$, не зависящие от $\ell$, такие, что при $\ell \geqslant 1$ справедливы неравенства

$$
\theta_{1} \frac{1}{\ell^{2}} \lambda_{\min }\left(A_{p}\right) \leqslant \lambda_{\min }\left(A_{0}\right) \leqslant \lambda_{\min }\left(A_{m}\right) \leqslant \theta_{2} \frac{1}{\ell^{2}} .
$$

Доказательству соотношений (8) посвящены следующие два раздела, а пока напомним, что все $\lambda\left(A_{p}\right)=1$ и $\gamma^{2}=\lambda_{\min }\left(A_{0}\right)$. 
3. Оценка сверху. Расмотрим в прямоугольной области $\Omega$ (4) следующую спектральную задачу $A_{m} p=\lambda p$ : найти собственные значения $\lambda$ и соответствующие собственные функции $p \in P$, удовлетворяющие системе уравнений

$$
-\Delta \boldsymbol{u}+\operatorname{grad} p=0, \quad \operatorname{div} \boldsymbol{u}=\lambda p,
$$

и модельному набору краевых условий для $\boldsymbol{u}=\left(u_{1}, u_{2}\right) \in \boldsymbol{U}_{m}$ :

$$
\begin{aligned}
& u_{1}=0, \quad u_{2}=0 \quad \text { при } x_{2}=0, L_{2} \text {, } \\
& u_{1}=0, \quad \frac{\partial u_{2}}{\partial x_{1}}=0 \quad \text { при } x_{1}=0, L_{1} \text {. }
\end{aligned}
$$

Задача (9)-(11) имеет аналитическое решение, которое может быть найдено методом разделения переменных (методом Фурье) [13]. Имеет место

Лемма 1. Oператор $A_{m}$ в задаче (9)-(11) имеет собственное значение

$$
\lambda\left(A_{m}\right)=\frac{1}{2}\left(1-\frac{t}{\operatorname{sh} t}\right), \quad \text { əде } t=\pi \frac{L_{2}}{L_{1}} .
$$

ДокаЗАтЕльство. Рассмотрим спектральную задачу $A_{m} p=\lambda p$ в области $\Omega=$ $\left(0, L_{1}\right) \times(-b, b)$ при $b=L_{2} / 2$. Этот сдвиг исходного прямоугольника $(4)$ не меняет собственных значений, но существенно облегчает проведение выкладок. Далее положим $r=\pi / L_{1}$ и непосредственной проверкой убедимся, что функции

$$
\begin{gathered}
u_{1}=\frac{1}{2} \sin r x_{1}\left[b \frac{\operatorname{sh} r b}{\operatorname{ch} r b} \operatorname{ch} r x_{2}-x_{2} \operatorname{sh} r x_{2}\right], \\
u_{2}=-\frac{1}{2} \cos r x_{1}\left[b \frac{\operatorname{ch} r b}{\operatorname{sh} r b} \operatorname{sh} r x_{2}-x_{2} \operatorname{ch} r x_{2}\right] \\
p=\cos r x_{1} \operatorname{ch} r x_{2}
\end{gathered}
$$

удовлетворяют соотношениям (9)-(11) вместе с указанным выше собственным значением. Лемма доказана.

ЛЕмма 2. Для любой области $\Omega$ прямоугольной формы имеет место неравенство

$$
\lambda_{\min }\left(A_{0}\right) \leqslant \lambda_{\min }\left(A_{m}\right) .
$$

ДокАЗАТЕЛЬСТво. Напомним функциональные определения нижних границ спектров операторов $A_{0}$ и $A_{m}$ :

$$
\lambda_{\min }\left(A_{0}\right)=\inf _{p \in P} \sup _{\boldsymbol{u} \in \boldsymbol{U}} \frac{(p, \operatorname{div} \boldsymbol{u})^{2}}{\|\boldsymbol{u}\|_{\boldsymbol{U}}^{2}\|p\|_{P}^{2}}, \quad \lambda_{\min }\left(A_{m}\right)=\inf _{p \in P} \sup _{\boldsymbol{u} \in \boldsymbol{U}_{m}} \frac{(p, \operatorname{div} \boldsymbol{u})^{2}}{\|\boldsymbol{u}\|_{\boldsymbol{U}}^{2}\|p\|_{P}^{2}}
$$

Теперь справедливость утверждения леммы следует из того факта, что $\boldsymbol{U} \subset \boldsymbol{U}_{m}$ (см. определения пространств в предыдущем разделе). Лемма доказана.

Сформулируем оценку сверху в неравенстве (8) в следующем виде. Имеет место 
Теорема 1. В рассматриваемой области $\Omega=\left\{\left(x_{1}, x_{2}\right) \mid 0<x_{i}<L_{i}, i=1,2\right\}$ справедлива равномерная по $\ell=L_{1} / L_{2} \geqslant 1$ оченка

$$
\lambda_{\min }\left(A_{0}\right) \leqslant \theta_{2} \frac{1}{\ell^{2}}
$$

с постоянной $\theta_{2}=\pi^{2} / 12$.

ДокАЗАТЕЛЬСТво. Из лемм 1 и 2 непосредственно следует цепочка неравенств

$$
\lambda_{\min }\left(A_{0}\right) \leqslant \lambda_{\min }\left(A_{m}\right) \leqslant \lambda\left(A_{m}\right)=\frac{1}{2}\left(1-\frac{t}{\operatorname{sh} t}\right),
$$

где $t=\pi L_{2} / L_{1}=\pi / \ell$. Далее для формулы в правой части после элементарных преобразований получаем

$$
\left(1-\frac{t}{\operatorname{sh} t}\right)=\frac{t^{2}}{2 \cdot 3 !} \frac{1+\frac{t^{2} 3 !}{5 !}+\frac{t^{4} 3 !}{7 !}+\cdots}{1+\frac{t^{2}}{3 !}+\frac{t^{4}}{5 !}+\cdots} \leqslant \frac{\pi^{2}}{12} \frac{1}{\ell^{2}}
$$

Теорема доказана.

4. Оценка снизу. Чтобы не загромождать изложение, в настоящем разделе рассуждения проводятся для прямоугольной области $\Omega$ вида $(0, \pi \ell) \times(0, \pi)$. Имеет место

ТЕОРема 2. В рассматриваемой области $\Omega$ справедлива равномерная по $\ell \geqslant 1$ оиенка

$$
\theta_{1} \frac{1}{\ell^{2}} \leqslant \lambda_{\min }\left(A_{0}\right)
$$

с постоянной $\theta_{1}=1 / 60$.

ДокАЗАтЕльство. Заметим, что искомая оценка непосредственно следует из справедливости следующего неравенства для произвольной функции $p \in P$ :

$$
\theta_{1} \frac{1}{\ell^{2}} \sup _{\boldsymbol{U}_{p}} \frac{(p, \operatorname{div} \boldsymbol{u})^{2}}{\|\boldsymbol{u}\|_{\boldsymbol{U}}^{2}} \leqslant \sup _{\boldsymbol{U}} \frac{(p, \operatorname{div} \boldsymbol{u})^{2}}{\|\boldsymbol{u}\|_{\boldsymbol{U}}^{2}}
$$

Действительно, переходя к точной нижней грани по всем $p \in P$, будем иметь в правой части (12) $\lambda_{\min }\left(A_{0}\right)$, а в левой - указанную вьше оценку в силу того, что $\lambda_{\min }\left(A_{p}\right)=1$ $\left(A_{p} \equiv E\right)$.

Поэтому представляется удобным доказать соотношение (12). Рассмотрим для этого полную систему ортонормированных функций из $P$ :

$$
\boldsymbol{\Xi}=\left\{\psi_{m}: \psi_{m}=d_{m} \cos \frac{m_{1} x_{1}}{\ell} \cos m_{2} x_{2}\right\}, \quad d_{m}=\frac{\sqrt{\left(1+\operatorname{sign} m_{1}\right)\left(1+\operatorname{sign} m_{2}\right)}}{\pi \sqrt{\ell}}
$$

где $m=\left(m_{1}, m_{2}\right)$ - мультииндекс, $m_{i}(i=1,2)$ могут принимать значения $0,1,2, \ldots$, при этом $\max \left\{m_{1}, m_{2}\right\}>0$.

Докажем сначала (12) для произвольных тригонометрических многочленов из $P$ степени $M$ с константой $\theta$, не зависящей от $M$. Для этого рассмотрим функцию $p$ следующего вида:

$$
p=\sum_{m=0}^{M} p_{m} \psi_{m}, \quad p_{0}=0, \quad \psi_{m} \in \Xi
$$


Напомним (см. п. 1), что верхняя грань в выражении

$$
\sup _{\boldsymbol{u} \in \boldsymbol{U}_{p}} \frac{(p, \operatorname{div} \boldsymbol{u})^{2}}{\|\boldsymbol{u}\|_{\boldsymbol{U}}^{2}}
$$

достигается на функции $\widehat{\boldsymbol{u}}=\Delta_{p}^{-1} \nabla p$. Заметим также, что $\operatorname{div} \widehat{\boldsymbol{u}}=p$ в силу эквивалентности $A_{p}$ тождественному оператору. В данном случае вектор-функцию $\widehat{u}=\left(\widehat{u}_{1}, \widehat{u}_{2}\right)$, соответствующую выбранной $p$, можно записать в виде

$$
\widehat{u}_{k}=\sum_{m=0}^{M} b_{m}^{k} p_{m} \phi_{m}^{k}, \quad k=1,2
$$

где

$$
\begin{aligned}
b_{m}^{1}=\frac{m_{1}}{\ell\left(m_{1}^{2} \ell^{-2}+m_{2}^{2}\right)}, & b_{m}^{2}=\frac{m_{2}}{\left(m_{1}^{2} \ell^{-2}+m_{2}^{2}\right)}, \\
\phi_{m}^{1}=d_{m} \sin \frac{m_{1} x_{1}}{\ell} \cos m_{2} x_{2}, & \phi_{m}^{2}=d_{m} \cos \frac{m_{1} x_{1}}{\ell} \sin m_{2} x_{2} .
\end{aligned}
$$

Поэтому для доказательства неравенства (12) достаточно построить такую $\boldsymbol{u} \in \boldsymbol{U}$, что следуюшие неравенства будут справедливы с некоторыми положительными постоянными $c_{1}, c_{2}<\infty$, не зависяшими от $M, \ell, \widehat{u}$ :

$$
(p, \operatorname{div} \widehat{\boldsymbol{u}})^{2} \leqslant c_{1}(p, \operatorname{div} \boldsymbol{u})^{2}, \quad\|\boldsymbol{u}\|_{\boldsymbol{U}}^{2} \leqslant c_{2} \ell^{2}\|\widehat{\boldsymbol{u}}\|_{\boldsymbol{U}}^{2}
$$

Для этого, рассмотрим следующие наборы индексов:

$$
M_{1}=\left\{m: m_{1} \geqslant m_{2}\right\}, \quad M_{2}=\left\{m: m_{1}<m_{2}\right\} .
$$

Заметим, что $M_{1} \cap M_{2}=\varnothing$ и для любого индекса $m$ существует $i \in\{1,2\}$ такое, что $m \in M_{i}$. Представим функцию $p$ в виде суммы двух ортогональных частей

$$
p^{(i)}=\sum_{m \in M_{i}} p_{m} \psi_{m}, \quad i=1,2 .
$$

Здесь и далее мы подразумеваем $p_{m}=0$ при $\max \left(m_{1}, m_{2}\right)>M$.

Так как $\operatorname{div} \widehat{\boldsymbol{u}}=p=p^{(1)}+p^{(2)}$, существует $i \in\{1,2\}$ такое, что

$$
\left|\left(p^{(i)}, p\right)\right| \geqslant \frac{1}{2}|(p, p)|=\frac{1}{2}|(\operatorname{div} \widehat{u}, p)| \text {. }
$$

Пусть неравенство (14) справедливо для $i=1$. Тогда определим вектор-функцию $\boldsymbol{u}=$ $\left(u_{1}, u_{2}\right) \in \boldsymbol{U}$ следующим образом:

$$
\begin{gathered}
u_{1}=\sum_{m \in M_{1}} \frac{\ell}{m_{1}} p_{m} \phi_{m}^{1}-\sum_{m} \frac{\ell}{m_{1}} \rho_{m}^{(1)} \phi_{m}^{1}, \quad u_{2} \equiv 0, \\
\text { иначе } \quad u_{2}=\sum_{m \in M_{2}} \frac{1}{m_{2}} p_{m} \phi_{m}^{2}-\sum_{m} \frac{1}{m_{2}} \rho_{m}^{(2)} \phi_{m}^{2}, \quad u_{1} \equiv 0,
\end{gathered}
$$

где коэффициенты $\rho_{m}^{(1)}$ и $\rho_{m}^{(2)}$ будут определены ниже. 
Для произвольного $m_{i}$ обозначим через $C\left(m_{i}\right)$ наименьшее четное целое число большее $m_{i}, i=1,2$. Если неравенство (14) справедливо для $i=1$, то произвольному индексу $m=\left(m_{1}, m_{2}\right)$ поставим в соответствие индекс $\bar{m}=\left(\bar{m}_{1}, \bar{m}_{2}\right)$, где $\bar{m}_{1}=m_{1}$, $\bar{m}_{2}=m_{2}\left(\bmod C\left(m_{1}\right)\right)$, а коэффишиенты $\rho_{m}^{(1)}$ определим по формулам

$$
\rho_{m}^{(1)}= \begin{cases}\frac{1}{3} p_{\bar{m}}, & \text { если } \bar{m} \in M_{1}, m_{2}<3 C\left(m_{1}\right), \\ 0 & \text { иначе. }\end{cases}
$$

Если женеравенство (14) справедливо для $i=2$, топроизвольному индексу $m=\left(m_{1}, m_{2}\right)$ поставим в соответствие индекс $\widetilde{m}=\left(\widetilde{m}_{1}, \widetilde{m}_{2}\right)$, где $\widetilde{m}_{2}=m_{2}, \widetilde{m}_{1}=m_{1}\left(\bmod C\left(m_{2}\right)\right)$, a коэффициенты $\rho_{m}^{(2)}$ определим по формулам

$$
\rho_{m}^{(2)}= \begin{cases}\frac{1}{3} p_{\tilde{m}}, & \text { если } \widetilde{m} \in M_{2}, m_{1}<3 C\left(m_{2}\right) \\ 0 & \text { иначе. }\end{cases}
$$

Из приведенных вьше построений следует, что $\boldsymbol{u}$ - тригонометрический многочлен степени не больше $3(M+2)$ и $\left.\boldsymbol{u}\right|_{\partial \Omega}=0$.

Оценим $\|\boldsymbol{u}\|_{\boldsymbol{U}}$ и $|(p, \operatorname{div} \boldsymbol{u})|$, при этом будем пользоваться тем, что системы функций $\left\{\phi_{m}^{1}\right\}$ и $\left\{\phi_{m}^{2}\right\}$ ортонормированы относительно скалярного произведения в $L_{2}$, а нормы функций $\boldsymbol{u}, \widehat{\boldsymbol{u}}$ и $p$ выражаются явно через соответствующие коэффициенты Фурье. Обозначим $\rho^{(i)}=\sum_{m} \rho_{m}^{(i)} \phi_{m}^{1}$ для $i=1,2$ и рассмотрим отдельно оба случая. В первом случае $-\bar{m} \in M_{1}$ и $5 m_{1} \geqslant m_{2}-$ имеем

$$
\begin{aligned}
\left\|\frac{\partial u_{1}}{\partial x_{1}}\right\|_{P}^{2} & =\left\|p^{(1)}\right\|_{P}^{2}-2\left(p^{(1)}, \rho^{(1)}\right)+\left\|\rho^{(1)}\right\|_{P}^{2}=\frac{1}{3}\left\|p^{(1)}\right\|_{P}^{2}+\left\|\rho^{(1)}\right\|_{P}^{2} \\
& \leqslant \frac{2}{3}\left\|p^{(1)}\right\|_{P}^{2} \leqslant \frac{2}{3}\|p\|_{P}^{2}=\frac{2}{3}\|\operatorname{div} \widehat{u}\|_{P}^{2} \leqslant \frac{2}{3}\|\widehat{\boldsymbol{u}}\|_{U}^{2} .
\end{aligned}
$$

Здесь мы воспользовались соотношениями

$$
\begin{gathered}
\left(p^{(1)}, \rho^{(1)}\right)=\sum_{m \in M_{1}} p_{m} \rho_{m}^{(1)}=\frac{1}{3} \sum_{m \in M_{1}} p_{m}^{2}=\frac{1}{3}\left\|p^{(1)}\right\|_{P}^{2} \\
\left\|\rho^{(1)}\right\|_{P}^{2}=\sum_{m}\left[\rho_{m}^{(1)}\right]^{2}=3 \sum_{m \in M_{1}} \frac{1}{9} p_{m}^{2}=\frac{1}{3}\left\|p^{(1)}\right\|_{P}^{2}
\end{gathered}
$$

Далее

$$
\left\|\frac{\partial u_{1}}{\partial x_{2}}\right\|_{P}^{2} \leqslant \ell^{2}\left[\left\|p^{(1)}\right\|_{P}^{2}-2\left(p^{(1)}, \rho^{(1)}\right)+\frac{35}{9}\left\|p^{(1)}\right\|_{P}^{2}\right]=\frac{38}{9} \ell^{2}\left\|p^{(1)}\right\|_{P}^{2} \leqslant \frac{38}{9} \ell^{2}\|\widehat{u}\|_{U}^{2} .
$$

Вьше мы воспользовались оценкой

$$
\begin{aligned}
\left\|\frac{\partial}{\partial x_{2}}\left(\sum_{m} \frac{\ell}{m_{1}} \rho_{m}^{(1)} \phi_{m}^{1}\right)\right\|_{P}^{2} & =\ell^{2} \sum_{m}\left(\frac{m_{2}}{m_{1}}\right)^{2}\left[\rho_{m}^{(1)}\right]^{2} \\
& \leqslant \ell^{2} \frac{1}{9} \sum_{m \in M_{1}}\left(1+3^{2}+5^{2}\right) p_{m}^{2}=\frac{35}{9}\left\|p^{(1)}\right\|_{P}^{2}
\end{aligned}
$$


Вспоминая, что $\left|\left(p, p^{(1)}\right)\right| \geqslant \frac{1}{2}|(p, \operatorname{div} \widehat{\boldsymbol{u}})|$, также получаем

$$
\begin{aligned}
|(p, \operatorname{div} \boldsymbol{u})| & =\left|\left(p, \frac{\partial u_{1}}{\partial x_{1}}\right)\right|=\left|\left(p, p^{(1)}-\rho^{(1)}\right)\right| \geqslant\left|\left(p, p^{(1)}\right)\right|-\left|\left(p, \rho^{(1)}\right)\right| \\
& \geqslant\left(1-\frac{2}{\sqrt{6}}\right)\left|\left(p, p^{(1)}\right)\right| \geqslant \frac{\sqrt{6}-2}{2 \sqrt{6}}|(p, \operatorname{div} \widehat{\boldsymbol{u}})| .
\end{aligned}
$$

Вьше мы воспользовались оценкой при $\varepsilon=\sqrt{3 / 2}$

$$
\begin{aligned}
\left|\left(p, \rho^{(1)}\right)\right| & =\frac{1}{3} \sum_{m=0}^{M} p_{m} p_{\bar{m}} \leqslant \frac{\varepsilon}{6} \sum_{m=0}^{M} p_{m}^{2}+\frac{1}{6 \varepsilon} \sum_{m=0}^{M} p_{\bar{m}}^{2} \\
& \leqslant \frac{\varepsilon}{3} \sum_{m \in M_{1}} p_{m}^{2}+\frac{1}{2 \varepsilon} \sum_{m \in M_{1}} p_{m}^{2}=\frac{2}{\sqrt{6}}\left|\left(p, p^{(1)}\right)\right| .
\end{aligned}
$$

Во втором случае $-\widetilde{m} \in M_{2}$ и $4 m_{2} \geqslant m_{1}$ - аналогичньг образом получаем

$$
\begin{gathered}
\left\|\frac{\partial u_{2}}{\partial x_{1}}\right\|_{P}^{2} \leqslant \frac{38}{9} \frac{1}{\ell}\|\widehat{\boldsymbol{u}}\|_{\boldsymbol{U}}^{2}, \quad\left\|\frac{\partial u_{2}}{\partial x_{2}}\right\|_{P}^{2} \leqslant\left\|p^{(2)}\right\|_{P}^{2}+\left\|\rho^{(2)}\right\|_{P}^{2} \leqslant \frac{2}{3}\|\widehat{u}\|_{\boldsymbol{U}}^{2}, \\
|(p, \operatorname{div} \boldsymbol{u})| \geqslant \frac{\sqrt{6}-2}{2 \sqrt{6}}|(p, \operatorname{div} \widehat{\boldsymbol{u}})| .
\end{gathered}
$$

Из неравенств (15)-(18) непосредственно следует, что оценки (13) справедливы с константами $c_{1}=12, c_{2}=5$ при условии $\ell \geqslant 1$. Так как $c_{1}$ и $c_{2}$ не зависят от степени многочлена $M$, неравенство (12) справедливо для всех конечных тригонометрических многочленов с константой $\theta=\left(c_{1} c_{2}\right)^{-1}$. А так как множество таких многочленов всюду плотно в $P$, переходя к замыканию в (12) мы доказываем теорему.

5. Основной результат. Оценки, полученные в предыдущих разделах, позволяют установить справедливость следующего утверждения. Имеет место

Теорема 3. Для областей $\Omega$ прямоугольной формы,$\Omega=\left\{\left(x_{1}, x_{2}\right) \mid 0<x_{i}<L_{i}\right.$, $i=1,2\}$, обозначим $\ell=\max \left(L_{1} / L_{2}, L_{2} / L_{1}\right)$. Тогда для константы $\gamma$ из

$$
\gamma=\inf _{p \in P} \sup _{\boldsymbol{u} \in \boldsymbol{U}} \frac{|(p, \operatorname{div} \boldsymbol{u})|}{\|\boldsymbol{u}\|_{\boldsymbol{U}}\|p\|_{P}}
$$

имеет место асимптотика $\gamma=O\left(\ell^{-1}\right), \ell \rightarrow \infty$.

ДокАЗАТЕЛЬСТво. При $\ell=L_{1} / L_{2} \geqslant 1$ из теорем 1 и 2 следуют равномерные по $\ell$ оценки

$$
\theta_{1} \frac{1}{\ell^{2}} \leqslant \lambda_{\min }\left(A_{0}\right) \leqslant \theta_{2} \frac{1}{\ell^{2}},
$$

откуда в силу равенства $\lambda_{\min }\left(A_{0}\right)=\gamma^{2}$ имеем искомую асимптотику.

Далее заметим, что для фиксированного прямоугольника одновременное сжатие (или растяжение) сторон с коэффициентом $\alpha \neq 0$ не меняет собственных значений оператора $A_{0}$. Это следует из замены независимых переменых в равенстве (3)

$$
\left(A_{0} q, q\right)=\sup _{\boldsymbol{u} \in \boldsymbol{U}} \frac{(q, \operatorname{div} \boldsymbol{u})^{2}}{\|\boldsymbol{u}\|_{\boldsymbol{U}}^{2}}
$$


с последующим сокращением числителя и знаменателя на $\alpha^{2}$. Кроме того, поворот прямоугольника на произвольньй угол $\varphi$ в плоскости $\mathbb{R}^{2}$ также приводит к аналогичному результату [11]. Отсюда немедленно следует, что для областей $(0, \ell) \times(0,1)$ и $(0,1) \times\left(0, \ell^{-1}\right)$ при любом $\ell$ множества собственных значений операторов $A_{0}$ совпадают. Таким образом, указанная асимптотика справедлива и в случае $\ell=L_{2} / L_{1} \geqslant 1$. Теорема доказана.

В заключение следует отметить, что вытянутые прямоугольники являются типичными вычислительными областями в математическом моделировании различных эффектов, связанных с движением жидкости (плазмы). Поэтому важньм практическим приложением полученного результата является получение количественной информации о том, насколько трудоемко будет нахождение численного решения даже линейных уравнений в длинных каналах.

\section{СПИСОК ЦИТИРОВАННОЙ ЛИТЕРАТУРЫ}

[1] Дьяконов Е. Г. Минимизация вычислительной работы. Асимптотически оптимальные алгоритмы для эллиптических задач. М.: Наука, 1989.

[2] Girault V., Raviart P. A. Finite element methods for Navier-Stokes equations. Berlin: Springer, 1986.

[3] Langer U., Queck W. On the convergence factor of Uzawa's algorithm // J. Comp. Appl. Math. 1986. V. 15. P. 191-202.

[4] Чижонков Е. В. К сходимости метода искусственной сжимаемости. // Вестн. МГУ. Сер. 1. Матем., мех. 1996. № 2. С. 13-20.

[5] Chizhonkov E. V. Application of the Cossera spectrum to the optimization of a method for solving the Stokes Problem // Russ. Journal of Numer. Analysis and Math. Modelling. 1994. V. 9. № 3. P. 191-199.

[6] Михлин С.Г. Спектр пучка операторов теории упругости. // УМН. 1973. Т. 28. №3. C. $43-82$.

[7] Cosserat E. et F. Sur la déformation infiniment petite d'une enveloppe sphérique élastique. // C. R. Acad. Sci. Paris. 1901. V. 133. P. 326-329.

[8] Chizhonkov E. V. On the Constant in the LBB condition for ring domains. Report No. 9537 (October 1995). The Netherlands: Dept. of Math. Univ. of Nijmegen, 1995.

[9] Crouzeix M. Etude d'une methode de linearisation. Resolution des equations de Stokes stationaries. Application aux equations des Navier-Stokes stationares // Cahiere de l'IRIA. 1974. № 12. P. 139-244.

[10] Михлин С. Г. Дальнейшее исследование спектра Коссера. // Вестн. ЛГУ. Сер. Матем. 1967. Т. 7. № 2. С. $96-102$.

[11] Кобельков Г. М. Об эквивалентных нормировках подпространств $L_{2}$ // Anal. Math. 1977. № 3. C. $177-186$.

[12] Ольшанский М. А. Задача Стокса с модельными краевыми условиями // Матем. сб. 1997. T. 188. № 4. C. 127-144.

[13] Aristov P.P., Chizhonkov E. V. On the Constant in the LBB condition for rectangular domains. Report No. 9534 (September 1995). The Netherlands: Dept. of Math. Univ. of Nijmegen, 1995.

Московский государственный университет им. М.В.Ломоносова

Поступило

07.10 .1997

Исправленный вариант

10.09.1999 